\title{
Modelling and Simulation for Energy Optimization of Refrigeration System
}

\author{
C.W. Chen, Y.W. Lin, F.Z. Chen \\ Instrument Technology Research Center \\ NARL \\ Taiwan
}

\begin{abstract}
A refrigeration system achieves its refrigeration function generally through cryogenic refrigerant generated by multiple refrigerant compressor units. Traditionally, the operation of refrigerant compressor units is controlled only by experience or the proportional-integral-derivative controller of refrigerant temperature or pressure. Due to the lack of scientific, systematic and optimum logic basis, it is difficult to optimize the operation of refrigeration system and control the excessive energy consumption with these methods. On this basis, this study employs the system optimization method to determine the optimum operation of each compressor unit within the system to obtain the most energy-saving operation for maximum operation efficiency of the refrigeration system (energy consumption efficiency), according to the actual required refrigeration capacity at load side, actual performance and operation condition of each unit. The results reflect that the system optimization method can obtain the optimum solution and increase the coefficient of performance of the original refrigeration system by $7.83 \%$.
\end{abstract}

Keywords-refrigeration system; coefficient of performance; energy optimization; modelling and simulation

\section{INTRODUCTION}

The penetration rate of refrigeration systems is regarded as an important indicator for the standard of living of a country. According to a UN investigation, in developing countries half the food has spoiled during shipping due to the lack of adequate refrigeration equipment, thus aggravating the food shortage. In developed countries, complete refrigeration equipment such as refrigerated and frozen storage ensures a better life. However, in addition to the high equipment cost, refrigeration systems have considerable power consumption and the refrigerated and frozen storage operates on electricity. According to a Japanese investigation, the power cost of refrigerated and frozen storage accounts for approximately $15 \%-20 \%$ of the total operation cost [8]. The previous design of refrigerated and frozen storage emphasized the storage quality and missed energy saving. In recent years, saving energy for refrigeration system while maintaining the quality of contents has gained attention of researchers in the refrigeration system industry due to energy shortage.

The refrigeration system must be designed to meet a range of frozen needs, particularly in Taiwan, which has four distinct seasons and refrigerated and frozen storage needs to adapt to various changes. The refrigeration system must operate in a permissible range by changing operation points so as to provide different refrigeration capacities, eventually satisfying different needs. Therefore, in common designs, multiple refrigerant compressor units are used to produce the required refrigeration in a 'flexible' manner.

Traditionally, the operation of refrigerant compressor units is controlled only by experience or proportional-integralderivative (PID) controllers of refrigerant temperature or pressure; the direct digital control (DDC) is used for setting and adjusting the operational status of each compressor in the system. Due to the lack of scientific, systematic and optimum logic basis, it is difficult to optimize the operation of the refrigeration system and control the excessive energy consumption with these methods. An advanced control method requires the establishment of an appropriate refrigeration system model by which the dynamic action of the system can be resolved and based on which the optimum control strategy can be designed to maintain the system at the optimum operating point. While researches on optimum control strategy are still few, due to the complexity of the refrigeration system, establishing a model for optimum operation control and solving it further still remains a subject of research.

The design introducing the optimization theory to refrigeration system control model can improve traditional control methods to enhance the energy efficiency and freezing quality. At present, in the field of air conditioning system, many researchers have studied the optimization design, operation and control method of chiller, air handling unit, icestorage air conditioning system and chiller plant to maximize benefits, save energy and achieve a comfort level [1] [2] [4] [9] [10] [11] [13] [14]. However, the progress has been slow, with little research literature, in the field of industrial refrigeration systems [3]. Taiwan is located in a tropical area, where vegetable, fruit and fish preservation relies greatly on refrigerated and frozen storage; therefore, optimizing the refrigeration system for best economy and reliability is a subject worthy of serious study.

Based on the above considerations, this study proposes a new control model to maximize the operation efficiency of the refrigeration system, i.e. energy consumption efficiency by using the system optimization technology, considering the actual required refrigeration capacity at load side as the basis of operation adjustment of all compressor units in the system and considering actual performance and operation condition of each unit. The model will determine the optimum operation mode for each compressor unit with its optimization logic and algorithm so as to achieve the optimum coefficient of performance (COP) of the refrigeration system when the 
refrigeration capacity of refrigerated and frozen storage is satisfied.

\section{ENERGY OPTIMIZATION CONTROL MODEL OF REFRIGERATION SYSTEM}

The optimum operating strategy goal of the refrigeration system is to control the operational status of each compressor unit so as to maximize the average COP after the system runs for some time and satisfies all operating restrictions. From the mathematical perspective, this control can be described as a system optimization control model. Figure 1 is a diagram of a typical refrigeration system operation. This study proposes a new control model 'energy optimization control model of refrigeration system' based on this operation framework to maximize operation (energy) efficiency of the refrigeration system, by employing the mathematical modelling optimization programming from system optimization method [5] [6] [12] and considering the refrigeration capacity actually requested by the refrigerated and frozen storage and true operating performance and operation conditions of each unit as demand restrictions. On this basis, the operational status of each compressor unit in the system is optimized, thus achieving the optimum system performance. The mathematical model is established as follows [5] [6] [12]:

\section{Objective Function:}

$$
\text { Max. COP }=\frac{\sum_{t=1}^{n} \sum_{j=1}^{m} v_{j}^{t} \times q_{j} \times O L R_{j}^{t}}{\sum_{t=1}^{n} \sum_{j=1}^{m} v_{j}^{t} \times p_{j}^{t}+v_{j}^{t} \times\left(1-v_{j}^{t-1}\right) \times s_{j}}
$$

\section{Subject to:}

$$
\begin{gathered}
\sum_{j=1}^{m} v_{j}^{t} \times q_{j} \times O L R_{j}^{t} \geq Q^{t}, t=1,2,3, \ldots, n \\
\left\{\begin{array}{cc}
O L R_{\mathrm{j}, \min }^{\mathrm{t}} \leq \mathrm{OLR}_{\mathrm{j}}^{\mathrm{t}} \leq \mathrm{OLR}_{\mathrm{j}, \mathrm{tmax}}^{\mathrm{t}} & \text { if } \mathrm{v}_{\mathrm{j}}^{\mathrm{t}}=1 \\
\mathrm{OLR}_{\mathrm{j}}^{\mathrm{t}}=0 & \text { if } \mathrm{v}_{\mathrm{j}}^{\mathrm{t}}=0 \\
, j=1,2,3, \ldots, m ; t=1,2,3, \ldots, n
\end{array}\right. \\
\left\{\begin{array}{cc}
p_{j}^{t}=f\left(O L R_{j}^{t}\right) & \text { if } v_{j}^{t}=1 \\
p_{j}^{t}=0 & \text { if } v_{j}^{t}=0
\end{array}\right. \\
j=1,2,3, \ldots, m ; t=1,2,3, \ldots, n \\
v_{j}^{t} \in[0,1], j=1,2,3, \ldots, m ; t=1,2,3, \ldots, n
\end{gathered}
$$

In equation (1), we set the objective function for the maximum COP of the refrigeration system. Equation (2) is a constraint in which we assume that for time stage $t$, the total operating capacity of each compressor unit meets the refrigeration capacity required by the refrigerated and frozen storage. Equation (3) is a constraint in which we assume that for time stage $t$, the load rate of each compressor unit conforms to the operating conditions of the unit. Equation (4) is a constraint in which we assume that for time stage t, the operating power of each compressor unit conforms to the load characteristics of the unit. Equation (5) is a constraint in which we assume that for time stage t, the operational status of each compressor unit can only be in two states, 'Start' and 'Stop'. The following are the symbol definitions. $t$ : time stage. We set the unit control period of the refrigeration system to $24 \mathrm{~h}$, which is divided into time stages of the same interval $(\Delta t=10 \mathrm{~min})$ as time discretization. Therefore, the unit control period of $24 \mathrm{~h}$ is divided into 144 time stages.

\section{$j$ : compressor unit.}

COP: operation (energy) efficiency indicator of the refrigeration system, represented generally as $\mathrm{RT} / \mathrm{Kw}$, i.e. refrigeration capacity (RT) generated by one unit of power demand $(\mathrm{Kw})$. The higher COP is better for the operation (energy) efficiency and energy conservation of refrigeration system. The unit is RT/Kw.

$v_{j}^{t}$ : operational status of compressor unit $\mathrm{j}$ for time stage $\mathrm{t}$ (0: stop; 1: start).

$q_{j}$ : rated refrigeration capacity (RT) of compressor unit $\mathrm{j}$.

$O L R_{j}^{t}$ : operating load rate (\%) of compressor unit j for time stage $\mathrm{t}$.

$p_{j}^{t}$ : operating power $(\mathrm{Kw})$ of compressor unit $\mathrm{j}$ for time stage $\mathrm{t}$.

$v_{j}^{t-1}$ : operational status of compressor unit $\mathrm{j}$ for time stage $\mathrm{t}-1$ (0: stop; 1 : start).

$s_{j}$ : loss of power $(\mathrm{Kw})$ when the operational status of compressor unit $\mathrm{j}$ is switched from 0 to 1 .

$Q^{t}$ : refrigeration capacity (RT) required by the refrigerated and frozen storage for time stage $t$.

$O L R_{j, \min }^{t}$ : lowest operating load rate (\%) of compressor unit $\mathrm{j}$ for time stage $\mathrm{t}$.

$O L R_{j, \text { max }}^{t}$ : highest operating load rate (\%) of compressor unit $\mathrm{j}$ for time stage $\mathrm{t}$.

$f\left(O L R_{j}^{t}\right)$ : function of the relationship between operating load rate and power of compressor unit $\mathrm{j}$ for time stage t. We assume that operating power of the compressor unit is a cubic polynomial function of load rate, namely, $p_{j}^{t}=f\left(O L R_{j}^{t}\right)=a_{j}+$ $b_{j} \times O L R_{j}^{t}+c_{j} \times\left(O L R_{j}^{t}\right)^{2}+d_{j} \times\left(O L R_{j}^{t}\right)^{3}$, where $\mathrm{a}_{\mathrm{j}}, \mathrm{b}_{\mathrm{j}}, \mathrm{c}_{\mathrm{j}}$ and $d_{j}$ represent the marginal power value of compressor unit $j$.

In conclusion, 'energy optimization control model of refrigeration system' can automatically determine a set of optimum start/stop $\left(v_{j}^{t}\right)$ and load rate $\left(O L R_{j}^{t}\right)$ status of each compressor unit in unit control time ( $24 \mathrm{~h} / 144$ time stages), at the refrigeration capacity required by refrigerated and frozen storage in a given period, to maximize COP in equation (1) and satisfy all constraints of equations (2)-(5). 


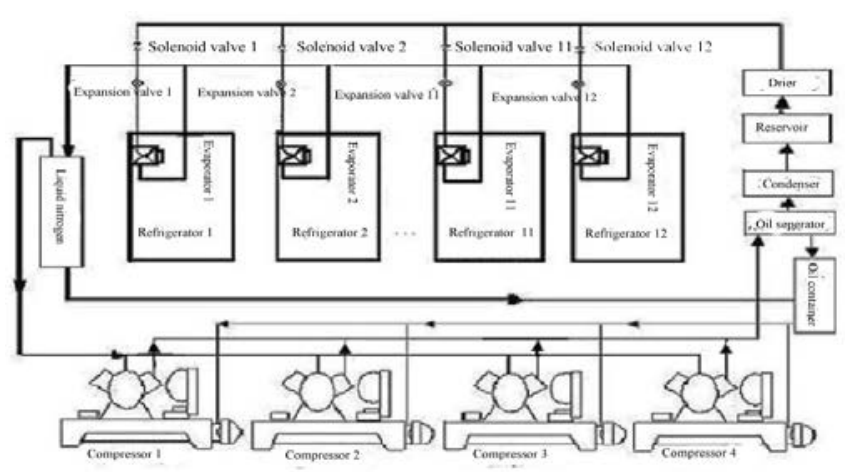

FIGURE I. OPERATION FRAMEWORK OF REFRIGERATION SYSTEM [7].

\section{CASE TEST AND ANALYSIS}

The example of this study is a refrigeration system with three 20RT scroll compressor units. The operational performance function and constraints refer to Table 1.

Substitute the operating parameters of each compressor unit into the 'energy optimization control model of refrigeration system' in Table 1 for the resolution with system optimization method after the refrigeration capacity required by refrigerated and frozen storage in each time stage is given. Thus, the optimum operating condition for each compressor unit (including start/stop status and load rate), and the optimum COP of refrigeration system will be easily obtained.

The study makes full use of LINGO PACKAGED SOFTWARE to complete the establishment and solving of the optimization model in Windows 7 operating system.

The work uses case refrigeration system actual refrigeration capacity (RT) and power demand $(\mathrm{Kw})$ at a certain period (01.June-2013 to 30.June-2013) to compare differences in average energy efficiency (COP) for the 'energy optimization control model of refrigeration system' and the current refrigeration system under equivalent refrigeration capacity and loss of power for each unit.

Figure 2 reveals the comparison results of COP before and after optimization of compressor units of 2013.06.012013.06.30 refrigeration systems. The '---' shows the actual COP from system actual refrigeration capacity generated by power demand per day, and the ' '...' shows the simulated COP from 'energy optimization control model of refrigeration system' under
TABLE I. OPERATIONAL PERFORMANCE FUNCTION AND CONSTRAINTS OF EACH COMPRESSOR UNIT.

Compressor unit \#1

$p_{1}^{t}=2.52+20.47 \times O L R_{1}^{t}-26.34 \times\left(O L R_{1}^{t}\right)^{2}+19.71$

$\times\left(O L R_{1}^{t}\right)^{3}$

$40 \% \leq O L R_{1}^{t} \leq 100 \%$;

$q_{1}=20$;

$s_{1}=10$;

Compressor unit \#2

$p_{2}^{t}=1.66+15.16 \times O L R_{2}^{t}-9.51 \times\left(O L R_{2}^{t}\right)^{2}+6.9$

$\times\left(O L R_{2}^{t}\right)^{3}$

$40 \% \leq O L R_{2}^{t} \leq 100 \%$;

$q_{2}=20$;

$s_{2}=10$;

Compressor unit \#3

$p_{3}^{t}=3.25+7.61 \times O L R_{3}^{t}+0.36 \times\left(O L R_{3}^{t}\right)^{2}+2.5 \times\left(O L R_{3}^{t}\right)^{3}$;

$40 \% \leq O L R_{3}^{t} \leq 100 \%$;

$q_{3}=20$;

$s_{3}=10$;

equivalent refrigeration capacity and loss of power for each unit with current refrigeration system. The average running COP prior to system optimization is $3.98 \mathrm{RT} / \mathrm{Kw}$. However, after optimization with 'energy optimization control model of refrigeration system' established in this study, the simulated result reveals that the average COP increases to $4.29 \mathrm{RT} / \mathrm{Kw}$ with an average energy saving rate of $7.83 \%$.

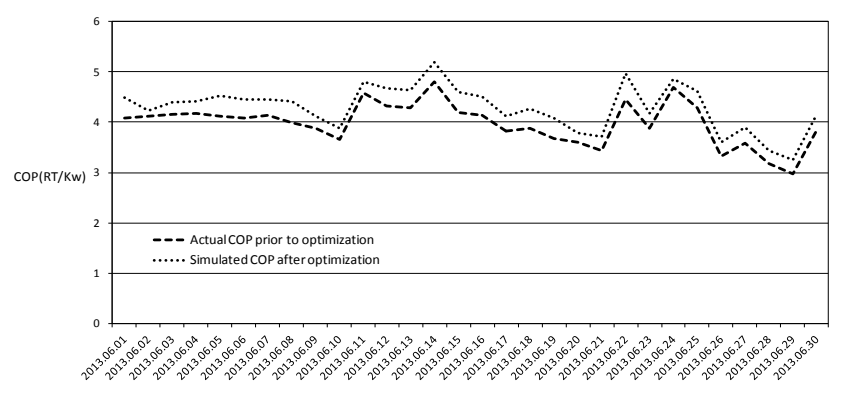

FIGURE II. COP COMPARISONS BEFORE AND AFTER SYSTEM OPTIMIZATION.

\section{SUMMARY AND CONCLUSIONS}

According to the simulation results, we observe that these results are consistent with our expectations and constraints; therefore, mathematical modelling optimization method can greatly promote the operational performance of the refrigeration system. The 'energy optimization control model of refrigeration system' can determine the optimum operational status of each compressor unit within the system (including unit start/stop status and load rate), without changing the existing compressor unit or influencing the refrigeration quality, i.e. maintaining an equal refrigeration ability, in order to obtain better energy efficiency (COP).

\section{REFERENCES}

[1] C.W. Chen, C.W. Lee and C.Y. Chen, (2010). To Enhance The Energy Efficiency of Chiller Plants With System Optimization Theory. Energy \& Environment, 21(5), 409-424. 
[2] J.E. Braun, J.W. Mitchell and S.A. Klein, (1987). Performance and Control Characteristics of a Large Cooling System. ASHRAE Transactions, 93(1), 1830-1852.

[3] J. Zhang and E.A. Groll, (2000). Saving Energy in Refrigerated Warehouses. ASHRAE Journal, 42(8), 35-39.

[4] M.A. Cascia, (1988). Optimizing Chiller Plant Energy Savings Using Adaptive DDC Algorithms. ASHRAE Transactions, 94(2), 1937-1946.

[5] M.S. Bazaraa, H.D. Sherali and C.M. Shetty, (1993). Nonlinear Programming Theory and Algorithms. John Wiley \& Sons Inc., U.S.A.

[6] M. Gen and R. Cheng, (2000). Genetic Algorithms and Engineering Optimization. John Wiley \& Sons Inc., U.S.A.

[7] R.J. Dossat, (1991). Principles of Refrigeration. John Wiley \& Sons Inc., U.S.A.

[8] S. Yoshio, K. Hajime, S. Kunio, K. Mimoru and I. Hiroshi, (1984). Management System of Cold Storage. Refrigeration, 59(684), 37-48.

[9] T.B. Hartman, (2005). Designing Efficient Systems with the Equal Marginal Performance Principle. ASHRAE Journal, 47(7), 64-70.

[10] T.B. Hartman, (2006). New Vistas with Relational Control. The Hartman Company Report, U.S.A.

[11] T.B. Hartman, (2007). Relational Control-21st Century More Effective Control Get More Effective System Performances. The Hartman Company Report, U.S.A.

[12] W.L. Winston, (1987). Operations Research: Applications and Algorithms. Duxbury Publisher, U.S.A.

[13] Y.C. Chang, (2004). A Novel Energy Conservation Method-Optimal Chiller Loading. Electric Power Systems Research, 69 (2-3), 221-226.

[14] Y.C. Chang, (2006). An Outstanding Method for Saving Energy-Optimal Chiller Operation. IEEE Transations on Energy Conversion, 21 March. 